\title{
Double Resonant Stub Bandstop Filter with Pseudo-Elliptic Response
}

\author{
J.A.G. Malherbe and C.A. Reid
}

\begin{abstract}
A simple wideband bandstop filter that yields equal ripples in both the pass and stop bands, is described. The filter comprises only of two resonant stubs and a connecting transmission line and has a transmission response almost identical to that of a third order elliptic function filter. The practical bandwidth is limited to $130-160 \%$.
\end{abstract}

Introduction: High rates of cutoff for wideband bandstop fitters are achieved if the filter prototype has a transfer function contributing transmission zeros at real frequencies away from the filter centre frequency, such as the elliptic function (Cauer) filters. The design procedures for transmission line filters employing elliptic function prototypes are widely described, and various forms of realization of such filters are available [1]. A number of authors have proposed and developed realizations of wideband bandstop filters that either realize true elliptic function responses, or pseudo-elliptic function responses by realizing additional transmission zeros at real frequencies.

In [2], a noncommensurate realization is described that is based on an elliptic function filter. Other authors add transmission zeros that are not related to the elliptic function prototype. In [3], a filter is described that is based on a conventional prototype such as Butterworth or Chebyshev, and to which additional transmission zeros are introduced by coupling the input and output lines. The split impedance ring filter of [4] contributes two additional zeros, while [5] describes a split impedance ring with three additional stubs. The physical structure of the filter in [6] resembles that of a third order elliptic function filter.

In this paper, the performance of a simple two-resonant stub filer is compared to that of a third order elliptic function filter and it is shown that a substantial reduction in complexity can be achieved for filters with relative bandwidths of between $130 \%$ and $160 \%$.

Filter Structure: Consider the microstrip centre conductor pattern shown in Fig. 1. The stub $Z_{1}$ resonates at the filter centre frequency of $f_{0}$, with $l_{0}=\lambda_{0} / 4$ while the stub $Z_{2}$ resonates at $f_{0} / 2$, length $2 l_{0}=\lambda_{0} / 2$. By separating the two stubs by a section of transmission line of $\lambda_{0} / 4$ and impedance $Z_{12}$ the proper phase relationship is introduced between the stubs, as well as to contribute additional attenuation in the stopband. Because $Z_{2}$ will again exhibit a resonance at $1.5 f_{0}$, a total of three transmission zeros occur on the real frequency axis between 0 and $2 f_{0}$, and equal ripples on either side of $f_{0}$ will result. 


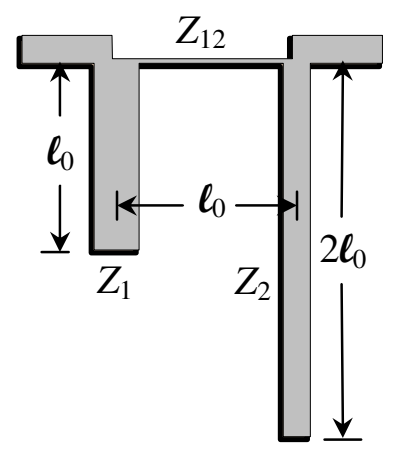

Fig. 1 Layout of the resonant stub filter.

The value of the unit element $Z_{12}$ is the main determinant of the filter bandwidth, which is limited to between $130 \%$ and $160 \%$; this is due to the fact that the positions of the transmission zeros are fixed. The bandwidth at the stopband attenuation level remains nominally at $110 \%$. The impedance values $Z_{1}$ and $Z_{2}$ in turn are the main determinants of the passband and stopband ripple levels. None of the values are sensitive, and acceptable combinations of bandwidth, passband and stopband ripple levels are readily obtained for realistic impedance levels through transmission line analysis of the structure.

Comparison with Elliptic Function Response: The response of a resonant stub filter with impedance values $Z_{1}=25 \Omega, Z_{2}=50 \Omega$, and $Z_{12}=190 \Omega$, is compared to the performance of a third order elliptic function filter designated C0302 with a passband ripple level of $0.0017 \mathrm{~dB}$ and stopband ripple level of $-21.6 \mathrm{~dB}$ from [7]; it is impedance scaled to $50 \Omega$ and frequency scaled to cut off at $\Omega^{\prime}=4.0$. By application of Richards' transform and two Kuroda transforms from the ports [1], the filter shown in Fig. 2 is obtained, with impedance values $Z_{1}=Z_{3}=81.7 \Omega$, $Z_{12}=Z_{23}=128.7 \Omega, Z_{21}=19.35 \Omega$, and $Z_{22}=18.66 \Omega$.

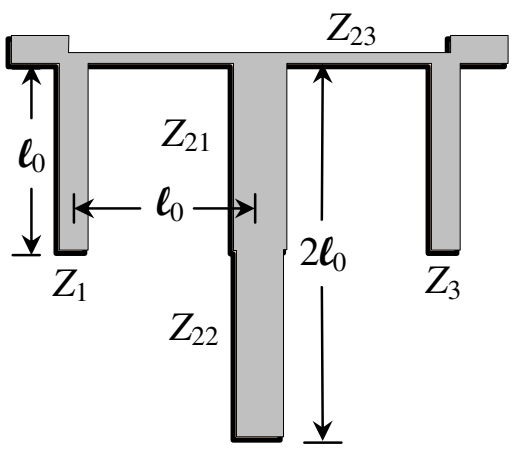

Fig. 2. Layout of the third order elliptic function filter.

Fig. 3 compares the responses of $S_{21}$ and $S_{11}$ for the two filters. They are clearly virtually identical. 


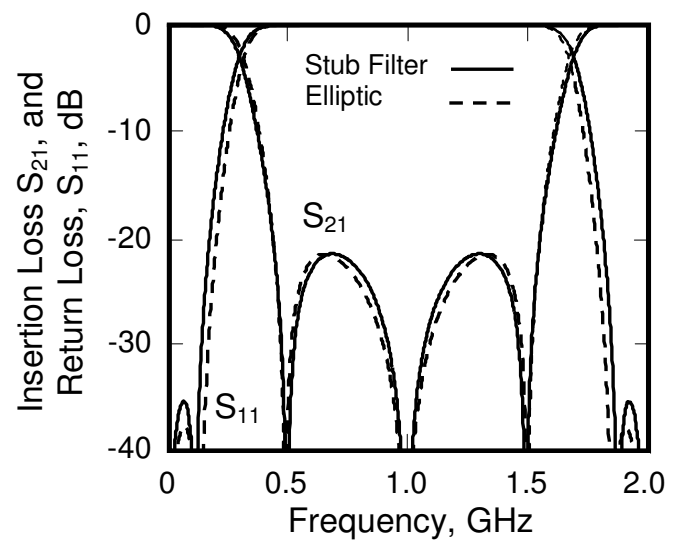

Fig. 3. Insertion loss and return loss of the resonant stub and the elliptic function filter.

Measured Results: A prototype filter was constructed in microstrip on RT Duroid 5880 with a dielectric thickness of $1.57 \mathrm{~mm}$, relative dielectric constant of 2.2 and loss tangent $\delta=0.0009$. The measured insertion loss and return loss is shown in Fig. 4. Both measured insertion and return loss show excellent agreement with the calculated values for the prototype filter.

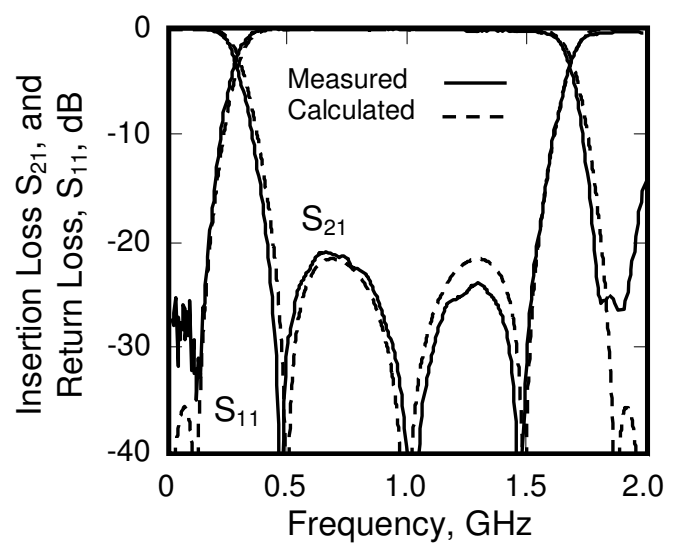

Fig. 4. Insertion loss and return loss of the constructed resonant stub filter.

Conclusion: The resonant stub filter exhibits a pseudo-elliptic function response that very closely resembles that of the true third order elliptic function filter, but which is only about half the size and complexity. The filter is simple to design, and filter is not sensitive to element values. Due to the transmission zeros at real frequencies being determined by the centre frequency of the filter, the applicable bandwidth is limited to between $130 \%$ and $160 \%$.

\section{REFERENCES}

[1] MALHERBE, J.A.G.: "Microwave Transmission Line Filters" (Artech House, Dedham, Mass. 1979). 
[2] MALHERBE, J.A.G.: "TEM Pseudo-Elliptic Function Filters Using Noncommensurate Lines", IEEE Trans. Microwave Theory Tech., 1976, 24(5), pp. 242-248.

[3] SHAMAN, H., and HONG, J-S.: "Wideband Bandstop Filter With CrossCoupling", IEEE Trans. Microwave Theory Tech.,2007, 55(7), pp. 17801785.

[4] MANDAL, M.K. and MONDAL, P.: "Design of sharp-rejection, compact, wideband bandstop filters", IET Microw. Antennas Propag., 2008, 2(7), pp. 389-393.

[5] DIVYABRAMHAM, K., MANDAL, M.K. and SANYAL, S.: "Sharp-Rejection Wideband Bandstop Filters", IEEE Microw. Wireless Compon. Lett., 2008, 18(10), pp. 662-664.

[6] VELIDI, K., GUNTUPALLI, A.B. and SANYAL, S.: "Sharp-Rejection UltraWide Bandstop Filters", IEEE Microw. Wireless Compon. Lett, 2009, 19(8), pp. 503-505.

[7] SAAL, R.: "Handbook of Filter design", (AEG-Telefunken, Berlin, 1979).

\section{Authors' affiliations:}

JAG Malherbe and C. Reid

Department of Electrical, Electronic and Computer Engineering

University of Pretoria

Pretoria 0002

South Africa

jagm@up.ac.za 